\title{
SENDEROS TRANSITAdOS EN LA CONSTRUCCIÓN DE UN IDEARIO INSTITUCIONAL PARA UNA MEJOR EdUCACIÓN SEXUAL INTEGRAL
}

\author{
Traced Paths in the construction of an institutional \\ ideology for a better Comprehensive Sexual Education
}

http://doi.org/10.33255/25914669/586

\begin{abstract}
Manuela Almirón
Jardín de Infantes N938, Dirección General de Cultura y Educación de la Provincia de

Buenos Aires. Universidad Nacional de Lanús, Argentina

manuelaunla@gmail.com https://orcid.org/0000-0001-7482-8423

Mónica Fernández Universidad Nacional de Lanús, Argentina

manerfernandez@gmail.com https://orcid.org/0000-0003-1025-2909
\end{abstract}

Recibido: 21 de septiembre de 2020

Aceptado: 30 de abril de 2021

\section{Resumen}

El artículo relata, a modo de crónica, la experiencia de un equipo de conducción del nivel inicial en la implementación del programa de Educación Sexual Integral (ESI). Esta institución ubicada en el conurbano de la provincia de Buenos Aires paralelamente estaba atravesando una situación particular de vinculación -revinculación- con la comunidad en la que se encuentra inserta y recibía el asesoramiento pedagógico-didáctico del proyecto de cooperación "Hablemos de derechos: La Educación Sexual Integral en las escuelas" de la Universidad Nacional de Lanús.

El contexto en que se produce esta experiencia es el de la implementación 
de la Ley 26.150, que desde su sanción en el año 2006 se volvió obligatoria en todos los establecimientos educativos de la República Argentina. Pero que sin embargo, su aplicación fue gradual y en la mayoría de los casos dilatada.

Palabas clave: Educación Sexual Integral - Comunidad - Equipo de trabajo

\section{Abstract}

The article chronicles the experience of an initial level leadership team in the implementation of the Comprehensive Sexual Education (ESI) program. This institution, located in the suburbs of the province of Buenos Aires, was simultaneously going through a particular situation of bonding -relinking- with the community in which it is inserted and received pedagogical-didactic advice from the cooperation project "Let's talk about rights: Education Comprehensive sexuality in schools" from the National University of Lanús.

The context in which this experience occurs is that of the implementation of Law 26150, which since its enactment in 2006 became mandatory in all educational establishments in the Argentine Republic. But nevertheless, its application was gradual and, in most cases, prolonged.

Key words: Comprehensive Sexual Education - Comunity - Team Work 


\section{Introducción}

"Se transforma la realidad porque este proceso de interacciones innovadoras requiere nuevas condiciones sociales, nueva distribución del poder y nuevos espacios para ir situando los retazos de nueva cultura que emergen en el aula. Como todo proceso de cambio conduce inevitablemente a enfrentamientos polémicos, dentro de una realidad plural cuyo desenlace. aunque imprevisible, será obviamente una modificación de la realidad" (Pérez Gómez, 2009, p. 44).

El presente artículo presenta el trabajo realizado en colaboración con el Proyecto de cooperación "Hablemos de derechos: La Educación Sexual en las Escuelas" de la Universidad Nacional de Lanús desde el ciclo 2018 al 2020 inclusive, con el Jardín de Infantes No 938 "Florencio Molina Campos" del distrito de Florencio Varela, de la Región Educativa $\mathrm{N}^{\circ} 4$.

El Jardín de Infantes No 938 "Florencio Molina Campos" es una institución pública de gestión estatal dependiente de la Dirección de Cultura y Educación de la Provincia de Buenos Aires que se encuentra ubicada en el Barrio Triángulo Don José de Florencio Varela en el límite con Claypole, Partido de Almirante Brown.

Para comprender la complejidad del territorio en que se emplaza la escuela es necesario mencionar que en el mismo se encuentran tres espacios ${ }^{1}$ bien diferenciados que se fueron conformando en distintas etapas: el Barrio Malvinas Argentinas II, el Triángulo de Don José y La Toma. El primero se construye con anterioridad al año 2000, es parte de un proyecto de zonificación local, el segundo se constituye a partir de la construcción de viviendas de material, linderas al antes mencionado y, por último, se encuentra el espacio conocido como La Toma, que se diferencia de los anteriores por ser un asentamiento conformado por viviendas precarias. El barrio tuvo un abrupto crecimiento en pocos años que se tradujo en la ocupación de espacios agrestes y públicos (como la plaza y el polideportivo). Puede decirse que las bases de la identidad barrial se ven atravesadas por severos conflictos que tienen lugar entre los pobladores originales y los nuevos habitantes que se fueron estableciendo en el lugar.

Si bien la iniciativa de solicitar la construcción de una escuela de nivel inicial en la zona surge de un grupo de vecinos y vecinas del Barrio Malvinas Argentinas II, el edificio escolar inaugurado en 2007 fue emplazado en el territorio correspondiente al Barrio Triángulo Don José. Así, el jardín viene a sumarse a una serie de instituciones barriales en las que confluye un clima de constante tensión: el polideportivo lindero al jardín que fue usurpado como la plaza que anteriormente había sido construida

1 Estos espacios además de ser habitacionales, son tres autodenominaciones barriales en un mismo barrio, un mismo territorio para el municipio. Tres etapas de urbanización de tierras al borde de las vías de tren que conformaron su propia cultura e identidad. 
por un grupo promotor de familias que participaban en el jardín, el Centro Integrador Comunitario Don José $(\mathrm{CIC})$ y dos escuelas primarias. La breve descripción realizada, común a la realidad de otras tantas comunidades del conurbano bonaerense, nos permite acercarnos a la complejidad en la trama de sus relaciones, cuyos conflictos escalan a severos niveles de violencia

El jardín cuenta con una matrícula de 180 estudiantes provenientes de los tres barrios mencionados y de otros de la localidad de Claypole, y muchas veces los conflictos entre los grupos vecinales se trasladan al espacio escolar. Dicha matrícula se encuentra dividida en seis secciones de 30 estudiantes cada una, distribuidos en dos turnos. El plantel docente está conformado por seis maestras, dos preceptoras (todas titulares), una secretaria, una directora y tres auxiliares.

Luego de esta breve descripción, en los siguientes apartados se desarrolla, el modo en que se tejieron los lazos comunitarios entres los espacios descriptos; los problemas y reflexiones que se suscitaron a partir de la puesta en práctica de las instancias de trabajo con la ESI. Nuestra experiencia también avanza en narrar la manera en que se construyen nuevas subjetividades que profesionalicen y habiliten, en el espacio institucional y comunitario, la enseñanza desde el paradigma de la diversidad. Intentamos también compartir de qué manera nuestra institución fue generando un registro que permitió hacer cortes evaluativos y re-direccionar las propuesta a fin sostener la práctica escolar desde el diálogo socio-comunitario.

\section{Los Lazos Comunitarios}

En el año 2013, se reconfiguró el vínculo entre comunidad y jardín, esto coincidió con la llegada del último asentamiento -La Toma-, lo que generó múltiples conflictos en la comunidad. Para describirlos tomaremos las categorías de Norbert Elías (2003) acerca de la relación entre los que denomina "establecidos" y "forasteros". Según este autor, muchas veces los problemas que subyacen en una comunidad son comunes a toda su población, las miradas de recelo entre partes hacen que los mismos sean atribuidos a quienes recién llegan, aun cuando son preexistentes. Los lazos de afecto entre las "antiguas familias del barrio" hacen que se adjudiquen y universalicen virtudes modélicas (como las del mejor vecino y vecina del barrio), dejando por fuera o pretendiendo la expulsión de los y las recién llegados/as, acusándolos/as, estigma mediante, en el sentido en que lo piensa Norbert Elías², de causar muchos males en el barrio (inseguridad, basurales, inestabilidad eléctrica, conflictos sanitarios y otros). Sin embargo, se presenta una doble direccionalidad del conflicto en la que se

2 "La estigmatización, en tanto aspecto de la relación entre establecidos y forasteros, viene a menudo asociada con un tipo de fantasía colectiva desarrollada por el grupo establecido. Refleja y, al mismo tiempo, justifica la aversión (el prejuicio) que sus miembros sienten hacia los miembros del grupo forastero" (Elías, 2003, p. 236). 
niega lo que irrumpe y lo ya establecido en la comuna. Paralelamente, entre estos grupos, se establecen vínculos a través de lazos comerciales.

El jardín y su personal, para nada ajeno a la situación, se vio atravesado e invadido por conflictos entre familias, especialmente por el contexto de inseguridad.

El 21 de septiembre de 2013, el incendio de un automóvil robado hizo que el jardín "estallara" al explotar el equipo de gas del vehículo incendiado. El mismo año, las docentes fueron asaltadas a la salida de la jornada laboral y la directora fue agredida en la puerta del establecimiento. Estos y otros sucesos violentos, fueron generando distancia entre el equipo de trabajo del jardín y la comunidad. Cuando las situaciones violentas se direccionaron hacia el personal de la institución se generó inestabilidad en el equipo de conducción: a lo largo de dos años hubo alternancia de personal e incluso períodos en los que la institución no tuvo directivos a cargo. No obstante, el plantel docente se mantuvo estable, con una ideología defensiva (Dejours, 1988), ${ }^{3}$ que le permitió afrontar las situaciones, pero que dificultó la apertura institucional y el diálogo con la comunidad. Debe mencionarse además que se cortaron los vínculos interinstitucionales y no se lograba concretar trabajo en red (se suspendió el trabajo en equipo con otras instituciones educativas y la articulación con la Escuela Primaria N68, se cortó el diálogo con la Unidad de Gestión Local [UGL], se perdió el contacto tanto con el Centro Integrador Comunitario [CIC Dón José] como con el club o el "envión").

A mediados de 2015, un nuevo equipo de conducción toma los cargos de dirección ${ }^{4}$ y secretaria según establece la Planta Orgánica Funcional. El primer propósito de gestión para este equipo de conducción fue construir un espacio de encuentro, redefinición del vínculo familias-escuela y uno de los ejes para reconfigurar este vínculo fue el trabajo con la ESI.

Esto coincidió con la organización de jornadas de capacitación masivas por parte del Ministerio de Educación de la Nación ${ }^{5}$ conjuntamente con la Dirección General de Cultura y Educación de la Provincia (DGCyE) dirigida a docentes del nivel inicial y primario. Cada capacitación consistía en la asistencia a tres jornadas presenciales y el trabajo individual y en equipos a través de una plataforma virtual del Ministerio provista para tal fin.

Los 2 primeros encuentros fueron consecutivos, se trabajó en la autorreflexión -primer día- y en la exploración del material y las propuestas del programa para

3 El equipo de maestras se acopló con representaciones muy similares respecto a la comunidad que le sirvió como mecanismo de acción, y le permitió sostenerse mutuamente ante el riesgo inminente que les representaban las inseguridades y amenazas externas.

4 Manuela Almirón, una de las autoras del presente escrito.

5 El Ministerio de Educación de la Nación conjuntamente con los Ministerios Provinciales de Educación, planificaron capacitaciones masivas sobre ESI en vistas de las dificultades observadas en la implementación del programa en todos los niveles y modalidades del sistema educativo a pesar de que habían transcurrido 6 años de la sanción de la ley que prevé su creación. 
el nivel -segundo día-. Se realizaron exposiciones y trabajos grupales con formato taller para reflexionar acerca de los principales temas abordados por la ESI, las principales líneas de trabajo y material para su implementación.

En estos encuentros fueron presentados los lineamientos curriculares de la ESI (2008) y se trabajó en relación con los diseños curriculares provinciales de modo que los/as participantes tuviesen herramientas para el armado de planificaciones y secuencias didácticas, siendo el propósito transversalizar los ejes de la ley en las distintas áreas. Entendemos la transversalización como:

"una dimensión organizadora e integradora del currículo [que] implica la inclusión de temas, programas, ejes o enseñanzas transversales, los cuales poseen una vertiente cognitiva y otra de índole afectiva, donde se conjugan conocimientos e información con el mundo de los valores, las decisiones, los sentimientos y las actitudes, además de las acciones [que] permite la construcción de un pensamiento crítico [...] que amplía el escenario educativo más allá de los contenidos académicos clásicos..." (Bravo de Nava y otros, 2007, p. 4).

La tercera jornada tuvo lugar quince días después. En las dos semanas que mediaban, los y las docentes debían realizar una aproximación diagnóstica en sus escuelas. En este ejercicio, se relevaron elementos formales y prescriptos de la práctica docente: proyecto institucional, planificaciones, otros proyectos existentes que aborden la temática. También se indago sobre elementos pertenecientes al denominado "Curriculum oculto" (Fernández Enguita, 1998) que incluye el relevamiento de imágenes, comunicaciones, espacios, así como comentarios informales de docentes y estudiantes; y situaciones propias del cotidiano escolar como los principales emergentes relacionados a la temática ESI propias de cada establecimiento.

A partir de la participación en esos espacios de capacitación, y de la reflexión que se realiza al interior del jardín, quedaron expuestas una serie de resistencias por parte del cuerpo docente, posiblemente fundados en temores ante eventuales reacciones de las familias en relación a la implementación de la ESI y en la escasa o nula experiencia formativa en temas de sexualidad. Finalmente, el diagnóstico realizado en la institución expuso situaciones que ameritaban la elaboración de un proyecto institucional: la presencia de prejuicios, la circulación de chistes misóginos y homofóbicos entre el personal y la estigmatización del otro. Palabras y acciones que ponían de manifiesto las inconsistencias entre el discurso del personal y sus actos, ya sea a nivel de las relaciones interpares como para con estudiantes y familias.

Finalmente, la capacitación proponía como actividad final la organización de una jornada institucional para iniciar el trabajo en el marco del programa de Educación Sexual Integral y la incorporación del mismo como eje transversal en el Proyecto Educativo Institucional. Para ello se tomaba como insumo de trabajo diagnóstico realizado. 0 sea, cada institución diagramó una jornada distinta en 
base a sus problemas y posibilidades.

El diagnóstico del equipo de conducción consideraba que dicho proyecto requería un abordaje integral y holístico, no un mero estado administrativo que cumpliese con lo previsto en la normativa nacional y provincial. Para ello era necesario repensarse como educadoras, compañeras de trabajo, y especialmente repensar a las infancias como destinatarias de nuestro accionar, definiendo colectivamente cuál era la educación "deseada".

La relación didáctica con los contenidos prescriptos de ESI era eludida frecuentemente. Ante esta situación, el equipo de conducción pidió que se registre y se documente en los proyectos, secuencias y unidades didácticas todo el trabajo vinculado con ESI. Es decir, los contenidos ESI, hasta ese momento evitados, tomaron el mismo estatus prescriptivo que cualquier otro. Una vez establecido el deber, se presentó el desafío didáctico del ¿cómo abordarlo? En ese punto se detectó otro lugar clave para la intervención: algunos/as docentes seguían pensando que las y los estudiantes eran "niños pequeños" y no infancias diversas.

Paralelamente, se generaron espacios para presentar cuáles eran los modos de enseñanza y los contenidos ESI a las familias. Para la gestión, estas situaciones fueron oportunidades estratégicas para hacer participar e implicar a la comunidad. Entre finales de 2015 y abril de 2016 se pidió a la comisión pro-cooperadora, que comenzaba a organizarse, que acompañara con la compra de material bibliográfico vinculado con ESI para las bibliotecas áulicas, como así también para la biblioteca docente. La primera adquisición de libros en mucho tiempo fue acordada, de modo que se instituyó una particular forma de cultura escolar. No eran una colección de libros más, eran libros con mirada de género ${ }^{6}$.

\section{3. ¿De quiénes son los derechos?}

La evaluación final del ciclo 2015, puso dos aspectos claros en el horizonte: por un lado, repensar las y los sujetos de aprendizajes como diversidades y, por otro, el vínculo entre la escuela y la comunidad. En muchas oportunidades, el vector del debate fue poner en tensión el enfoque de derechos. En un primer momento el derecho de cada niño y niña que asiste al jardín, pero también de las personas adultas que transitan el espacio institucional. Como cuerpo docente, hubo una habilitación a a conocer la singularidad de cada historia, comprendiendo el espacio comunitario con el que se trabajaba, no solo las fachadas del barrio, sino el difícil conurbano

6 Se compraron colecciones infantiles (Colección "Mi cuerpo y yo", de Editorial Chicos.Net, Colección Antiprincesas, de Editorial Chirimbote; Colección "Ulises y Julieta", de Editorial Chicos. Net y otros que no respondían a colecciones). También, un Libro de asesoramiento docente, poniendo en manifiesto que las docentes también leían y construían nuevos modelos de enseñanza. Además, se compraron fascículos de enciclopedias para acercar a los niños al conocimiento científico propuesto por los lineamientos de la ESI. 
profundo ${ }^{7}$, con historias que resultaban y resultan dolorosas.

Dentro del trabajo con el equipo docente se tornó central la reflexión sobre los modos de nombrar a las y los otros/as y, también, a nosotros/as mismos/as. Poner en tensión qué pensamos cuando hacemos referencia acerca de los demás, reconfigurar ideas estandarizadas sobre las familias, que además habilitó un espacio para que la diversidad se nos presentara como oportunidad de repensar nuestra propia identidad.

Este trabajo introspectivo logró que, poco a poco, las familias dejaron de estar invisibilizadas 0 excluidas y fueron puestas en el eje de los debates que el cuerpo docente y auxiliar se daba. Para brindar un ejemplo de ello, se relata una situación suscitada en el ciclo lectivo 2017, cuando en una reunión con familias se dialogó sobre la Educación Sexual Integral y un padre dijo que "no todos los hombres eran malos", entonces las mismas familias respondieron y dieron el debate. En un primer momento, hubo un intercambio acalorado y algunas masculinidades se mostraron ofendidas. Sin embargo, las docentes tomaron esa escena para incorporar el concepto de género y más precisamente el de violencia de género. En aquella ocasión se vislumbraron en la comunidad dos cosas: por una parte, la posibilidad de las familias de expresarse y abrirse a relatar su sentir y por otro, que esos debates podían tener lugar en el espacio institucional. En poco tiempo, el vínculo con la comunidad dio un giro, comenzaba a participar activa y auténticamente en el jardín. Una comunidad de aprendizaje no puede presentarse escindida de las familias.

A medida que se sucedían los encuentros, aun cuando pudieran ser discordantes, se abrió un diálogo entre barrio y jardín. La propuesta iba más allá de lo que sucedía en las salas, había una preocupación por la integralidad de la ESI que aún no se podía traducir en palabras, pero que tenía que ver con democratizar el espacio institucional a partir de la integración con el barrio. Las reflexiones de Pérez Gómez auxiliaron para pensar estas transformaciones:

"Todo aprendizaje relevante es en el fondo un proceso de diálogo con la realidad social y natural o con la realidad imaginada. En la escuela se aprende una cultura socialmente seleccionada y la interacción con la misma será productiva y relevante desde el punto de vista educativo, cuando el alumno/a se introduzca en un proceso de diálogo creador con la misma, aceptando y cuestionando, rechazando y asumiendo. Este diálogo creador requiere, en nuestra opinión, una comunidad democrática de aprendizaje, abierta al contraste y la participación real de los miembros que la componen, hasta el punto de aceptar que se cuestione su propia razón, las normas que rigen los intercambios y el propio

7 El barrio tenía un espacio de casas construidas por un plan de viviendas (establecidos) y otro gran espacio con tierras tomadas (forasteros), que se fue urbanizando poco a poco -y aún no del todo- en el que no había acceso al agua corriente o a las cloacas, el acceso inicial al tendido eléctrico era por instalaciones clandestinas, no había calles trazadas por lo que a las viviendas se accedía por pasillos y las casas en general eran de madera. 
diseño del curriculum. Una comunidad democrática de aprendizaje, donde el conocimiento, las relaciones sociales, la estructura de las tareas académicas, los modos y criterios de evaluación y la propia naturaleza y función social de la escuela acepten someterse al escrutinio público de los estudiantes y docentes y a las consecuencias de sus reflexivas determinaciones" (Pérez Gómez, 1992, p. 113).

La dinámica escolar, en símisma, hace que muchos aspectos, proyectos, evaluaciones y criterios se adopten en una gestión colectiva, no siempre armónica o sincrónica. La posibilidad de sumar voces al proyecto educativo fue la intención del equipo de conducción, ya sea desde la apertura de nuevos debates en reuniones de familias como en los modos de atención al público ${ }^{8}$, pasando por la posibilidad de armar una cooperadora o un club de madres 9 .

Demás está decir que esta relación dialéctica entre la institución y la comunidad no fue homogénea. En el caso de las docentes, algunas manifestaron su desagrado por la opinión o la injerencia de las familias en la tarea pedagógica y en el manejo institucional. Tampoco todas las familias comprendieron o celebraron el estímulo a su participación de manera uniforme.

Para finalizar este apartado, se relata otro ejemplo, en el año 2017, también en situación de reunión con familias en la que se presentaba el personal y algunos proyectos del año, un padre dijo "este es un jardín feminista, pero eso está bien". Nuevamente, la respuesta no fue unidireccional se dialogó entre quienes se encontraban presentes. Docentes y comunidad reflexionando sobre ello. Algunas familias manifestaban que les parecía bien, otras que les parecía mal otras explicaban lo que el feminismo "es". Desde el equipo de conducción, nunca se había postulado a la institución como un "jardín feminista" por lo que el comentario causó sorpresa en el personal y provocó reflexiones puertas adentro. Tal vez, desde la comunidad este era el reflejo de los cambios que se iban dando.

Muchas veces, en la relación con la comunidad, se propone un estatus particular a los equipos docentes. Una "relación ética", como refiere Isabelino Siede (2017), en la que las familias le confieren confianza dado que el mismo rol hace que el

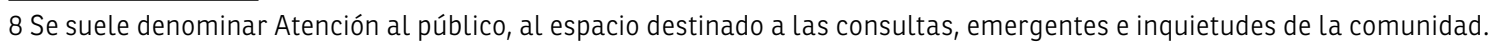

9 El Reglamento de las Instituciones Educativas promueve la creación de "Asociaciones de Padres", en el Reglamento General de Jardines de infantes se sugieren "Asociaciones de amigos del Jardín de infantes" y en otras reglamentaciones se nombra el "Club de Madres" como posibilidad. Cuando el Equipo de conducción se encuentra sin Comisión en la Cooperadora (y así sin acceso a las cuentas del Banco Provincia) convoca a la comunidad. Cuando se comienzan a reunir, eran madres las que se ofrecían a hacer tortas para vender, confeccionar juguetes o buscar algún familiar que pudiera cortar el pasto del gran predio. Al buscar una figura legal que enmarcara dichas actividades, se charló con la inspectora a cargo en ese momento y la sugerencia fue hacer el club de madres.

Con el tiempo, se dialogó con quienes participaban (y traccionaban a que otras vinieran también con equipos de mate a ser parte del espacio). No había varones en ese espacio, pero sí disidencias y familiares que no necesariamente eran "madres" de nuestros estudiantes. Por un tiempo, se autodenominaron Club de Madres aun cuando hubiera otras participantes. Con ellas, se comenzó la organización de la Asociación Cooperadora, cuando sí comenzaron a participar masculinidades. 
docente dé lo mejor en pos del cuidado y la enseñanza de quien tiene a cargo. Por eso mismo, el valor de la palabra de cada docente en las reuniones con familias cobra tanta importancia.

El trabajo de socialización e implicación comunitaria respecto a la enseñanza de la ESI fue sistemático y recurrente. Los miembros de la comunidad que iban transitando por el jardín daban cuenta de ello. En las reuniones con las familias esto se hacía explícito. Sin embargo, no se puede inferir que por estas situaciones anecdóticas la construcción de acuerdos y sentidos fuera homogénea. Ya lo advierte Siede,

"La participación de los grupos familiares en la deliberación sobre cuestiones institucionales implica un proceso de fuerte democratización, en tanto los reconoce como actores responsables por las decisiones que conciernen a los niños y niñas que están a su cargo. Esto no necesariamente implica que esa participación adopte un contenido democrático, pues en muchas ocasiones, al abrir las compuertas a la opinión familiar, algunas voces más conservadoras adoptan banderas ultramontanas que se resisten a las innovaciones que una institución propone, con fundamento técnico pedagógico" (Siede, 2017, p. 246).

Dentro de las opiniones y demandas que surgían de parte de las familias, llegaron al establecimiento voces que renegaban de un proyecto que desconocían. Algunas familias, tal vez motivadas por campañas mediáticas o preconceptos religiosos, se oponían radicalmente a las acciones que ya se encontraba en marcha, demandaban explicaciones. El equipo docente pudo poner en palabras qué, cómo y por qué se enseñaba lo que se enseñaba. No sin preguntar(se) una y otra vez a quién se le enseñaba, para qué se le enseñaba o cuáles eran los derechos que orientaron los caminos a adoptar.

Era evidente que había objetivos y propósitos que muy difícilmente se comenzaban a plasmar en proyectos. Pero esto generó sensaciones de tranquilidad, a partir de la necesidad de establecer valores en común que orientaran la intencionalidad educativa y brindaran un clima de trabajo que pueda sostenerse. No ya como un grupo cualquiera, sino como un equipo de trabajo. Entonces se trasladaron las inquietudes, más allá de los límites de nuestra comunidad. Comenzó la articulación con la Universidad Nacional de Lanús, específicamente con el equipo que coordina el Proyecto "Hablemos de Derechos. La Educación Sexual Integral en las escuelas"10, que cuenta entre sus propósitos el acompañamiento a instituciones en la revisión de programas y proyectos escolares en relación a la incorporación de los lineamientos curriculares del Programa ESI.

10 Proyecto de cooperación "Hablemos de Derechos: La Educación Sexual Integral en las escuelas. Expediente N² 2323/17 Universidad Nacional de Lanús. 
El diálogo permanente con el contexto, el entramado vinculante en el que el jardín comenzaba a ser parte de la búsqueda de construcción de caminos alternativos y miradas críticas que aporten soluciones, junto a la dilucidación de nuevos problemas fue reconstituyendo el vínculo con la comunidad. Es decir, al momento que el Jardín se implicaba en las situaciones de escala social y barrial, se fue inmiscuyendo en algunos modos de construir prácticas educativas emancipatorias. Esto se implementó como una manera de abordar la enseñanza en el marco de un entorno cercano en conflicto permanente. Pero fue a través del diálogo con el equipo de coordinación del proyecto de cooperación de la UNLa cuando se tomó real conciencia de ese aspecto, enseñando para quebrar lógicas de exclusión, matrices de dominación coloniales y patriarcales.

\section{Gestión de lo invisible, avances y nuevos problemas}

La propia práctica puertas adentro, debía ser reflexiva y cooperativa, la comunidad era coparticipe ${ }^{11}$, la supervisión acompañaba ante ciertos embates de la comunidad cuando se presentaban. La deconstrucción era la tarea y la gestión se propuso colaborar con ello, situar y contextualizar, promover un análisis del habitus práctico-reflexivo ${ }^{12}$.

Revisar la gestión de los espacios desde la ESI promovió movimientos y aperturas donde deconstruir los cuerpos y las corporalidades en el espacio escolar. Los espacios se encontraban diseñados y habilitados para que los cuerpos los transitaran con rigidez (Virilio, 1999). Aun cuando se contaba con espacios amplios y accesibles, no había salidas al aire libre, mucho menos salidas educativas en el barrio, por temores que se fundaban en que el barrio "tenía" ladrones y tiroteos .

Las propuestas de enseñanza y los juegos que incorporaban la ESI se compartieron y comunicaron a la comunidad, ya no en reuniones entre personas adultas, sino que contaban con la activa participación de las infancias. En los actos patrios se mostraba el trabajo desde una óptica que hacía visible la presencia de mujeres y rescataba sus voces. En los talleres con familias se promovió una mirada crítica sobre los roles de género y se socializaban estos modos de jugar con las niñas y los niños. Esto tuvo impacto en las charlas de las familias, tanto en la institución y fuera de ella ${ }^{13}$. El

11 Algunas organizaciones, no necesariamente educativas, se acercaban a la institución para generar acuerdos y proyectos: El polo productivo Don José, gestionado en ese entonces por Barrios de Pie, se ofreció a hacer cortes de césped para evitar el basural; la ONG Conciencia Ciudadana mantuvo diálogos en pos de poder realizar un mapeo barrial en el cual las familias pudieran visibilizar conflictos barriales que se encontraban naturalizados.

12 Antes del corredor seguro organizado en 2015 y vigente hasta 2016, fueron frecuentes tiroteos al horario de la "siesta". Dichos tiroteos retornaron en 2017 y en ocasiones se dieron en la puerta del jardín. En una oportunidad, ante un operativo de las fuerzas de seguridad fue necesario resguardar a estudiantes lejos de las ventanas

13 Así como algunas familias se ponían en juego dentro del jardín, también propusieron celebraciones del día del niño jugando en la 
respeto por la diversidad implica mayor libertad y eso no se dio sin debate.

El efecto fue rápidamente recuperado e hizo visible las infancias que comenzaban a manifestarse en ese entorno de mayor libertad. Los espacios que antes estaban estructurados en forma rígida y binaria, se pudieron rediseñar con suficiente flexibilidad para que inviten y habiliten la participación lúdica. Diversas niñeces interpelaban a docentes y familias poniendo en palabras sus intereses y sus sueños, rompiendo con la heterocisnorma o el binarismo.

El equipo docente se replanteó el concepto de género y sexualidad en la infancia luego de encontrarse con historias de vida que ponían en tensión lo preestablecido. Por ejemplo, durante los 3 años en que un estudiante ${ }^{14}$ asistió al jardín, solía expresar su incomodidad ante los compañeros varones, su grupo de referencia era preferentemente de niñas; jugaba con disfraces, manifestando su deseo de usar vestidos (lo que disgustaba a su padre, pero era legitimado por su abuela, quien le había regalado unas "can can" rosadas para el frío con la condición de que las usara bajo los pantalones). Esos momentos lúdicos permitieron expresar fantasías, narraba que le gustaría esperar a su marido con vestido en una casa similar a la de la maqueta del jardín. El juego permitió recuperar eso que posiblemente se estaba constituyendo, esas realidades no dichas en el entorno familiar que se ponían de manifiesto y se habilitaban en el contexto escolar.

En un primer momento, como equipo se intentó "definir" qué quería decir, pero luego se comenzó a pensar que el género puede fluir y más que poder establecer un nombre a lo que le sucedía se debían brindar las oportunidades para que esta persona se sintiera libre..$^{15}$

Situaciones como estas fueron de mucho debate y aprendizaje para el cuerpo docente. El equipo docente fue testigo privilegiado de ver estudiantes que invitaron a sus padres a jugar con muñecas, y luego ver a esos mismos padres cuestionar a la institución y finalmente comprender, en el diálogo con docentes, que en lo cotidiano las tareas de cuidado son de las familias, no de las feminidades.

A partir de 2017, la crisis impactó con fuerza en el orden escolar. Reaparecieron violencias de todo tipo, nuevamente sucedieron robos a docentes, inseguridad,

calle. El Centro Integrador Comunitario Don José hacía eco de nuestras actividades y comenzaba a articular actividades conjuntas para el cuidado de la salud y la prevención de la violencia contra las mujeres.

14 Una aclaración pertinente resulta de la reflexión sobre el uso de lenguaje inclusivo en este apartado. Si bien se pudiera haber enunciado el caso como "une estudiante" se considera que ello hubiera obturado en detrimento de los debates y posicionamientos adoptados institucionalmente. Es decir, se propiciaron espacios en los que el sujeto pudiera manifestarse, se brindaron espacios de afectividad, cuidado y libertad (un espacio donde advenir), pero de ninguna manera sería el equipo docente quien enuncie de modo diferente al que el sujeto dijera de sí mismo. Es por evitar un rótulo que en este caso no se utiliza el lenguaje inclusivo, no porque se responda a un mandato de masculinidad. 
vandalismo, falta de empleo, falta de acceso al gas -generalizado en el barrio-, desbordes cloacales, crisis sanitaria y cada vez más estudiantes con hambre. A lo que se sumó el recorte en el cupo del Servicio Alimentario Escolar (SAE).

A pesar de ciertos momentos de malestar, en el equipo docente supo continuar con la tarea y contener, también en esta ocasión, a la comunidad que atendía. En ese contexto las docentes dieron continuidad a la reflexión acerca de la educación, no se puede transitar un equipo de trabajo sin pensar(se) y construir casi de modo permanente. La percepción manifestada era de "tironeo" constante que en ocasiones las sumergía en pesimismo y desazón, porque el resto del tiempo la certeza de estar creando y consolidando espacios de educación social se había hecho carne y salían a dar respuestas. ${ }^{16}$ Para el equipo de trabajo eso también era ESI: enseñar a tener derechos aun cuando faltan, derecho a la dignidad, sobre todo. Esta tarea se realizó a partir de un trabajo que desdibujó fronteras entre los muros de la escuela y "el afuera", lo que permitió una vinculación en/ entre y con la comunidad, tal como se propone a partir de los ejes de lo que entendemos como pedagogía social. Porque:

"pensar la pedagogía social en nuestro tiempo, requiere incluir en el análisis los límites que ubican a los sujetos dentro de las categorías sociales inclusiónexclusión; con lo que se nos impone situar nuestras reflexiones en un territorio particular (...) se nos hace crucial contextualizar la transmisión de la cultura a las nuevas generaciones, en un proceso social- histórico particular y dentro de sistemas estatales cuyas políticas económicas, sociales y culturales han derivado en desigualdades sin parangón en la distribución material y simbólica de bienes a los ciudadanos" (Ceresani, Bugarín, Canciano, García, 2011, p. 7).

La búsqueda de "ser" en el barrio dio muchas idas y vueltas, pero siempre fue una meta del equipo de conducción. La historia da cuenta de una institución estallada (literalmente, en una explosión) y vandalizada muchas veces. Generar pertenencia en el barrio hizo que muchos proyectos tuvieran ese objetivo.

Los ciclos 2017 y 2018 fueron momentos de puertas adentro. El jardín se encontró promoviendo nuevamente proyectos para estimular la convivencia, la expresión de las emociones y secretos ${ }^{17}$. En este período, se dio continuidad a la articulación que se venía realizando con la Universidad.

En el ciclo 2019, en una reunión plenaria, surgió la idea de retomar aquel proyecto en el que el barrio caminaba conjuntamente por determinados sectores

16 Se realizaron reuniones con agentes municipales y de las fuerzas de seguridad, se articularon campañas de vacunación y de revisión del estado general de salud coordinadamente con el CIC Don José. 
para hacerlos más seguros. La CORRE-CAMINATA FAMILIAR es la propuesta de abordaje para la semana de las familias. La única consigna fue que todos y todas pudieran participar en familia. Los artífices del reglamento fueron las y los niños de las Terceras secciones. ${ }^{18}$ La premisa era simple, tan simple que por momentos las personas adultas no la comprendían: tienen que poder participar todos y todas, y todas las personas participantes ganarían. De ese modo, la reflexión escolar de la diversidad de familias se puso en escena: no importaba si eran 2 ó 20, las familias tenían que corre-caminar juntas y sin perder al equipo.

Como resultado, toda la comunidad educativa "anduvo" por el barrio: maestras, inspectoras y familias. Se pusieron en juego diversas estrategias para intentar llegar primero. El resumen de la jornada, acompañada de una merienda saludable y con la participación del equipo de salud del CIC Don José, fue la premiación para todas y todos. Y como cierre una clase abierta de zumba. Las palabras de cierre hicieron referencia a la enseñanza por el derecho a la dignidad, a un barrio transitable y una comunidad que pudiera acompañar en ello a las infancias. Los cuerpos estaban en la calle, esos cuerpos acallados en la rigidez impuesta o tullidos, se abrían camino, desde cochecitos de bebés a las abuelas con bastón pasando por posibilidades variopintas, todos en un regocijo de libertad.

La evaluación de este último proyecto dejó un eco, se trabajaba la Educación Sexual Integral no solo en un proyecto o una clase, se estaba haciendo de modo transversal y permanente. Esta iniciativa se abordó desde distintas áreas de aprendizaje, se trabajó por etapas y nos sirvió para ver el fruto de lo que hacíamos desde la ESI dentro y fuera del aula. Edith Litwin plantea que:

"...integrar significa que los estudiantes doten de sentido al conocimiento adquirido. Esto es, que reconozcan su origen, su valor y su vinculación con otros temas o problemas. Pueden relacionarlo con el pasado histórico, con intereses sociales, con necesidades personales. En síntesis, integrar es, desde la perspectiva del conocimiento, relacionar" (Litwin, 2016, p. 73).

Y en el análisis se llegó a otra certeza: la ESI no sólo debía ser transversal y sino también permanente. ${ }^{19}$ Pues, si solo se decía transversal, se escurría una parte y la ESI se encontraba en todas partes. La ESI tan temida era posible, estaba en la cultura institucional.

18 El reglamento decía que se debía "correr o caminar" con quien se "considerara parte de la familia", también que "todos iban a ser ganadores" pero que tenían que "llegar juntos a la meta como equipo familiar".

19 Mientras se discutía institucionalmente si era o no transversal surge un cuestionamiento inquietante ¿cómo sería un día en el jardín sin pensar en la ESI? ¿Sería igual como algo que ya se hizo cotidiano? ¿Y si fuera al revés, podría haber un día sin ESI? ¿Qué perjuicios traería? Entonces conjeturamos que no podíamos suponer la ESI como transversal si esta no era permanente. 
A lo largo de los años, hubo otros proyectos institucionales, también planificaciones áulicas, y sin programarlo se constituyó una cultura institucional particular. Cuando se llegó a ver con claridad que la Educación Sexual Integral ya estaba muy presente, surgió una nueva era necesario un proyecto específico.

En las tradiciones didácticas, los proyectos como estructura didáctica, se suelen utilizar cuando se diagnostica un problema o una carencia. En este caso se hacía contradictorio. Avanzando en el pensamiento, se entendió que no se requería un producto final. Hubo claridad en la idea de no disfrazar los propósitos pedagógicos de la institución. El dilema fue trasladado al equipo de la Universidad con el que se articulaba, y de manera conjunta y con expresa autorización de las autoridades de supervisión, se comenzó a pensar en un ideario institucional.

Previamente a los sucesos más complejos que se dieron en el 2020 por la pandemia, la institución se vio redactando dispositivos remotos y buscando, con el apoyo del proyecto de cooperación, tener la aprobación de las instancias superiores de supervisión para reemplazar los tradicionales proyectos por un ideario que diera cuenta de cada una de las estrategias desarrolladas hasta el momentoy de los acuerdos generados colectivamente. En este ideario se registraba la empatía puesta en diálogo con las familias, especialmente en el contexto de ASPO-DISPO, la valoración hacia los repertorios culturales de todos y cada uno de quienes pertenecen o transitan por la institución, la valoración de la diversidad en todas sus manifestaciones y el respeto por las diferentes formas de crianza, pero garantizando el cuidado. Las diversas infancias, la garantía y promoción de sus derechos, primordialmente el juego, la dignidad, el bienestar y la importancia de la enseñanza en entornos de libertad, que promuevan juegos y juguetes no sexistas, evitando los colores o los roles de género. En esta línea, se busca promover espacios de apertura y socialización con la comunidad toda. Se establece institucionalmente que las sanciones o llamados de atención también deben ser tenidos en cuenta como parte de la intervención desde la ESI y se hace hincapié en la promoción de vínculos afectivos cuidadosos, que retomen las manifestaciones emotivas sin prejuzgamientos. Respecto al cuidado de los cuerpos se propone la enseñanza sistemática pero también colaborativa con las familias, sobre todo en temas sensibles como el uso del baño. Además, se establece un apartado sobre las actividades cotidianas el cuidado en el uso de los distintos espacios y los proyectos establecidos institucionalmente que portan ya con una mirada de género. Se propone generar espacios de desmitificación de la ESI con las familias promoviendo comunidades democráticas de aprendizaje. Finalmente, se pregona la valoración propia y el cuidado amoroso de cada quien.

\section{Consideraciones Finales}

La sistematización de esta experiencia en una descripción cronológica ha permitido analizar las situaciones vivenciadas, para que el equipo pedagógico pueda volver 
sobre ellas de manera crítica. Al leerlas, han surgido aportes y visiones personales sobre cada suceso. El análisis da cuenta que cuando el equipo de conducción logró establecerse se pudo avanzar, no solo, en la transversalización de la Educación Sexual Integral.

De la descripción de los ejemplos y experiencias llevadas adelante se desprende el trabajo transdisciplinar, los avances hacia un diálogo sostenido que ha hecho partícipe a la comunidad de actividades pedagógicas. Es importante señalar que uno de los focos propuestos por la Ley de ESI es la articulación con las familias y otras instituciones. Una arista, muchas veces, poco trabajada que en este resultó ser la principal estrategia desarrollada.

La simetría en el diálogo y la construcción de los saberes no se podría haber suscitado sin el cambio profesional que posicionó al equipo docente como un cuerpo que entiende a las infancias como diversidades. Al respecto, el sostenimiento de este enfoque fue acompañado permanentemente por la Universidad (UNLa).

Parte de lo virtuoso de esta experiencia está vinculado a la importancia que tiene el trabajo conjunto, hacia el interior de la institución y con otras instituciones. La necesidad de planificar y escribir otras opciones a los tradicionales proyectos, hacer en ellos cortes evaluativos desde los cuales volver a planificar y especialmente escribir y dejar registro de cada una de las acciones que se desarrollan. Todo ello es el insumo vital para seguir trabajando.

\section{Bibliografía}

Bravo de Nava, E.; Inciarte González, A. y Febres Cordero M. (2007). La transversalidad como vía para la formación integral. Ponencia presentada en VII Reunión Nacional de Currículo y I Congreso Internacional de Calidad e Innovación en Educación Superior. Caracas, Venezuela. Web. [Fecha de consulta: 19 de abril de 2021] Disponible en: http:// saber.ucv.ve/ojs/index.php/rev_arete/article/download/21451/144814487744

Ceresani, V y otros (2011). Pedagogías en la formación de los educadores sociales. Web. [Fecha de consulta: 19 de abril de 2021] Disponible en: https://repositoriosdigitales. mincyt.gob.ar/vufind/Record/SEDICI_0c098524074e2ff02laab9clfbdcc14c

Cols, E. y Basabe, L. (2007). "La enseñanza" (pp. 125-162) En: Camilloni, A. (compilador). El saber didáctico. Ciudad Autónoma de Buenos Aires, Argentina: Ed. Paidós

Dejours, CH. (1988). Trabajo y desgaste mental: Ensayo de psicopatología del trabajo. Lima, Perú: Flora Tristán - Centro de la mujer peruana.

Elías, N. (2003). Ensayo acerca de las relaciones entre establecidos y forasteros. REIS, (pp. 219-251) Web. [Fecha de consulta: 19 de abril de 2021] Disponible en https:// dialnet.unirioja.es/descarga/articulo/837509.pdf 
Fernández Enguita, M. (1990). La cara oculta de la escuela. Educación y trabajo en el capitalismo. Madrid, España: Editorial Siglo XXI.

Giroux, H. (1990). Los profesores como intelectuales: hacia una pedagogía crítica del aprendizaje. Barcelona, España: Paidós

Litwin, E. (2016). El oficio de enseñar: condiciones y contextos. Ciudad Autónoma de Buenos Aires, Argentina: Ed. Paidós.

Pérez Gómez, A. (1992). Enseñanza para la comprensión En: Comprender y transformar la enseñanza. Madrid, España: Morata.

Pérez Gómez, A. (2009). Las funciones sociales de la escuela: de la reproducción a la reconstrucción crítica del conocimiento y la experiencia. Ciudad Autónoma de Buenos Aires, Argentina: LPP-CLACSO.

Sen, A. (2000). Desarrollo y libertad. Ciudad Autónoma de Buenos Aires, Argentina: Ed. Planeta.

Siede, I. (2017). Entre familias y escuela: alternativas de una relación compleja. Ciudad Autónoma de Buenos Aires, Argentina: Ed. Paidós.

Streck, D. (2015). Diccionario: Paulo Freire. Lima, Perú: CEAAL.

Virilio, P. (1999). La inseguridad en el territorio. Ciudad Autónoma de Buenos Aires, Argentina: La Marca.

Winnicott, D. (1971). Realidad y Juego. Barcelona, España: Ed. Gedisa 